\title{
Characterization and mitigation of coherent-optical-transition-radiation signals from a compressed electron beam
}

\author{
A. H. Lumpkin \\ Fermilab, Batavia, Illinois 60510, USA
}

\author{
N. S. Sereno, W. J. Berg, M. Borland, Y. Li, and S. J. Pasky \\ Argonne National Laboratory, Argonne, Illinois 60439, USA \\ (Received 23 January 2009; published 13 August 2009)
}

\begin{abstract}
The Advanced Photon Source (APS) injector complex includes an option for rf photocathode (PC) gun beam injection into the $450-\mathrm{MeV} \mathrm{S}$-band linac. At the $150-\mathrm{MeV}$ point, a four-dipole chicane was used to compress the micropulse bunch length from a few ps to sub-0.5 ps (FWHM). Noticeable enhancements of the optical transition radiation (OTR) signal sampled after the APS chicane were then observed as has been reported in the Linac Coherent Light Source (LCLS) injector commissioning. A far-infrared (FIR) coherent transition radiation detector and interferometer were used to monitor the bunch compression process and correlate the appearance of localized spikes of OTR signal ( 5 to 10 times brighter than adjacent areas) within the beam-image footprint. We have performed spectral-dependency measurements at $375 \mathrm{MeV}$ with a series of bandpass filters centered in 50-nm increments from 400 to $700 \mathrm{~nm}$ and with an imaging spectrometer and observed a broadband enhancement in these spikes. Mitigation concepts of the observed coherent OTR, which exhibits an intensity enhancement in the red part of the visible spectrum as compared to incoherent OTR, are described.
\end{abstract}

DOI: 10.1103/PhysRevSTAB.12.080702

PACS numbers: 41.60.Ap, 41.60.Cr

\section{INTRODUCTION}

The generation of ultrabright electron beams for various accelerator-based applications often involves compression of a photocathode (PC) rf gun beam using a four-magnet chicane configuration [1-3]. It was recognized via simulations that such beams would be susceptible to microbunching instabilities $[4,5]$ due to coherent synchrotron radiation (CSR) and linac wakefield effects. This was extended to effects of longitudinal space charge (LSC) by Saldin et al. [6], and a suppression scheme based on a laser heater was first proposed [5]. The detailed implementation of a laser heater on the Linac Coherent Light Source (LCLS) was described several years ago by Huang et al. [7]. However, all of these studies generally targeted microbunching with noticeable gain factors at wavelengths in the far infrared (FIR) at 8 to $12 \mu \mathrm{m}$ and greater. The scaling of the gain factor on intrinsic fractional energy spread in a onedimensional model shows an exponential suppression at shorter wavelengths [8]. There were issues of concern related to beam quality and reduced free-electron laser performance. A second issue became more apparent during the commissioning of the LCLS injector in 2007, when unexpected enhancements of the signals in the visible light optical transition radiation (OTR) monitors occurred after compression in a chicane bunch compressor [9]. These signals were attributed to a LSC-induced microbunching (LSCIM) that started from noise with a concomitant generation of coherent OTR (COTR) at the Al screens [8]. The strong enhancements at random locations and intensities within the beam-image footprint precluded OTR-based measurement of beam profiles, emittance, and slice energy spread at LCLS above beam energies of $135 \mathrm{MeV}$ and after the compressor $[9,10]$.

The Advanced Photon Source (APS) injector complex includes a flexible chicane bunch compressor [11] that is similar to that at LCLS and an option to use an rf photocathode (PC) gun. Since we also had previous experience with SASE-induced microbunching (SIM) [12], we decided to perform a series of experiments to explore the LSCIM-related phenomena. We initially performed studies on OTR measured at three screens located after the bunch compressor. We used focus-at-the-object or near-field imaging optics and established that there were clear enhancements of the OTR signals at maximum bunch compression. The compression was monitored with an FIR coherent transition radiation (CTR) monitor and interferometer. The shortest bunches generally generate the strongest FIR signals, and the appearance of the enhanced OTR was strongly correlated with the maximum FIR signal. However, there appeared to be a slight L2 phase setting difference for the FIR CTR and COTR intensity maxima. We also accelerated the compressed beam to the end of the linac and evaluated the enhancements at $\sim 375 \mathrm{MeV}$. The localized spikes in the beam distribution were not only still visible, but even stronger at this energy. At this latter station the light is transported outside of the tunnel to a small optics lab that allowed us to perform additional spectral-dependency measurements. Moreover, the use of a thermionic cathode (TC) gun pulse train with lower 
charge per micropulse was used for comparison purposes when the bunch length was compressed comparably to that of the PC gun beam. Discussions of the possible mechanisms for enhancing OTR signals will be presented for the APS case which is similar, but not identical to that of LCLS. A set of mitigation options for the COTR will be described which complement the expected effects of a laser heater [7].

\section{EXPERIMENTAL ASPECTS}

The tests were performed at the APS facility which includes an injector complex with two rf thermionic cathode (TC) guns for injecting into an S-band linac that typically accelerates the beam to $325 \mathrm{MeV}$, the particle accumulator ring, the booster synchrotron that ramps the energy from 0.325 to $7 \mathrm{GeV}$ in $220 \mathrm{~ms}$, a booster-tostorage-ring transport line, and the $7-\mathrm{GeV}$ storage ring. In addition, there is an rf photocathode (PC) gun that can also be used to inject into the linac as shown schematically in Fig. 1. A summary of the beam properties is provided in Table I. In addition, the beam energy spread at the end of the linac is about $0.1 \%$ for the PC rf gun and is generally larger for the TC rf gun, depending on the scraper position in the alpha magnet. The TC gun generates a series of micropulses with S-band frequency, and a kicker is used to only allow an 8-ns macropulse comprising 23 micropulses to be injected into the linac. The PC gun generates a single micropulse with more charge and lower emittance than those of the TC gun and at $6 \mathrm{~Hz}$ nominally. An extensive diagnostics suite is available in the chicane and after the chicane area (also shown in Fig. 1). The tests were performed in the linac at the three imaging stations (indicated by a flag symbol) after the chicane bunch compressor and at the end of the linac where another beam imaging station is located. A Michelson interferometer [13] which used a FIR coherent transition radiation (CTR) detector (Golay cell) is located just before the third screen of the first three-screen emittance station. A vertical bend dipole and diagnostics screens in this short beam line allow the monitoring of transverse $x$ beam size and energy following compression.

An Al-coated mirror with an $18 \mathrm{~mm}$ diameter was used to generate CTR, and it is mounted with its surface normal at 45 degrees to the beam direction on a pneumatic actuator assembly. A synthetic quartz lens at the port of the cross collimates the beam before the interferometer box. A remotely controlled translation stage steps the position of one arm of the interferometer for the autocorrelation measurements, and an EPICS interface allows the acquisition of the autocorrelation data. For the CTR intensity measurements the interferometer is set to the zero position. The

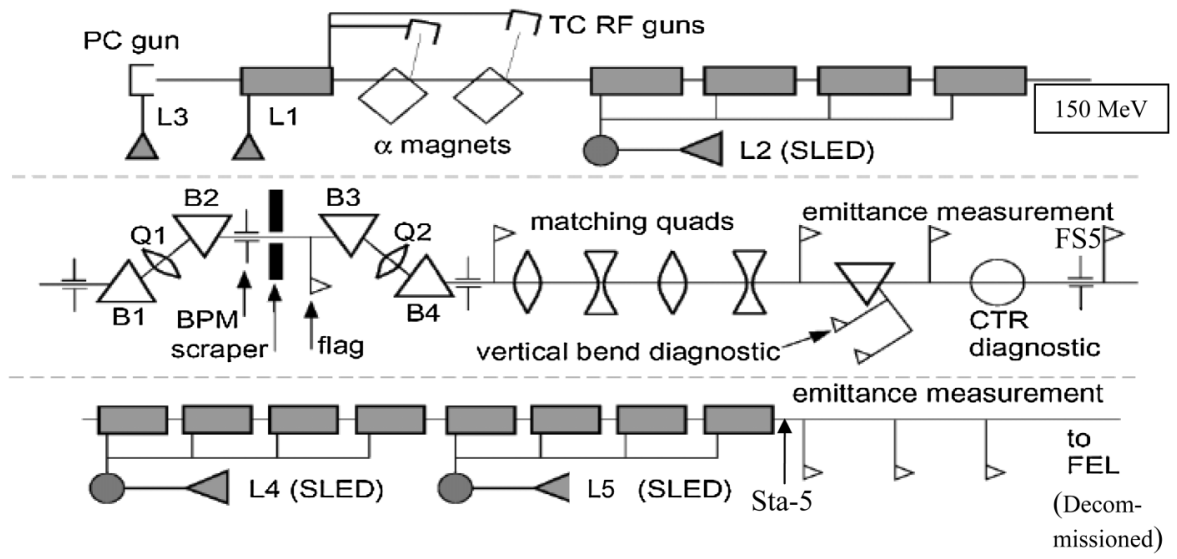

FIG. 1. A schematic of the APS linac test area showing the PC rf gun, the two TC rf guns, the accelerator structures (L1-L5), the chicane dipoles (B1-B4), and the diagnostics suite. This latter includes rf beam position monitors (BPMs), beam profile screens (flags), FIR CTR diagnostic, and the vertical bend diagnostic line. There is also an electron spectrometer located at the end of the linac just before Sta-5.

TABLE I. A summary of the electron beam properties for the APS TC $\mathrm{rf}$ gun 2 and the PC rf gun.

\begin{tabular}{lcc}
\hline \hline Parameter & TC rf gun beam & PC rf gun beam \\
\hline Charge per micropulse [pC] & $40-70$ & $300-500$ \\
Rms bunch length [fs] & $200-400$ & $300-400$ \\
Peak current [A] & $40-100$ & $300-600$ \\
Number of micropulses @ 6 Hz & 23 & 1 \\
Normalized emittance [ $\mu \mathrm{m}]$ & $10-12$ & $6-8$ \\
\hline \hline
\end{tabular}


beam profile screens consist of options for either a YAG:Ce crystal whose surface is normal to the beam direction with an Al-coated mirror at 45 degrees behind it or an Al OTR foil which can be inserted into the beam.

The light emitted from either radiator was directed to an analog CCD camera by turning mirrors and relay optics. These stations in the chicane area also have options for low- and high-resolution imaging of the beam spot by selecting one of two lens configurations [14]. The nearfield, low-resolution magnification configuration has calibration factors of $48.8 \mu \mathrm{m}$ per pixel in $x$ and $38.9 \mu \mathrm{m}$ per pixel in $y$. The incoherent OTR signal strength from a 400$\mathrm{pC}$ micropulse from the PC gun was too low to be usable with the analog CCD camera in the high-resolution mode. The signal intensity was adjusted with a remotely controlled iris (with no absolute position readback) in the path to the camera. The OTR and YAG:Ce images were recorded with a Datacube MV200 video digitizer for both online and offline image analyses, and a video switcher was used to select the relevant camera signal for digitizing. A normal image intensity integral was taken over a region of interest of all channels within a fixed radius from the peak position. A suitable background subtraction was also done in the offline analysis. Some plots are based on single row or column profiles as stated.

At the end of the linac, the imaging station designated Sta-5 includes the optical transport of the visible light out of the tunnel to a small, accessible optics lab where a CCD camera is located. This allows access for exploring the spectral dependency of the enhanced OTR. A set of bandpass filters with center wavelengths in 50-nm increments from 400 to $700 \mathrm{~nm}$ and 40-nm bandwidth as well as a 500$\mathrm{nm}$ short pass filter and a 500-nm long pass filter were used in the tests. In addition, an imaging spectrometer was installed with options for CCD camera or Pulnix ICCD camera readout. The beam energy was $325-375 \mathrm{MeV}$ at this station. The two detector options are somewhat complementary since the CCD chip with a glass cover plate covers about 380 to $700 \mathrm{~nm}$ with a peak sensitivity at $550 \mathrm{~nm}$, and the GaAs photocathode of the microchannel plate intensifier for the ICCD has almost constant sensitivity from 525 to $880 \mathrm{~nm}$.

Data were also recorded from the electron spectrometer at the end of the linac located just before Sta-5, but a Chromox screen was used at the focal plane of the spectrometer.

\section{INITIAL CTR AND OTR RESULTS}

The experiments were initiated by transporting the PC gun beam accelerated to $150 \mathrm{MeV}$ with a micropulse charge of 300-500 pC to the chicane area. The rf phase of the L2 accelerator structure located before the chicane was used to establish the appropriate conditions for compression in the chicane. The degree of compression was tracked with the Golay-cell signals (with the interferometer

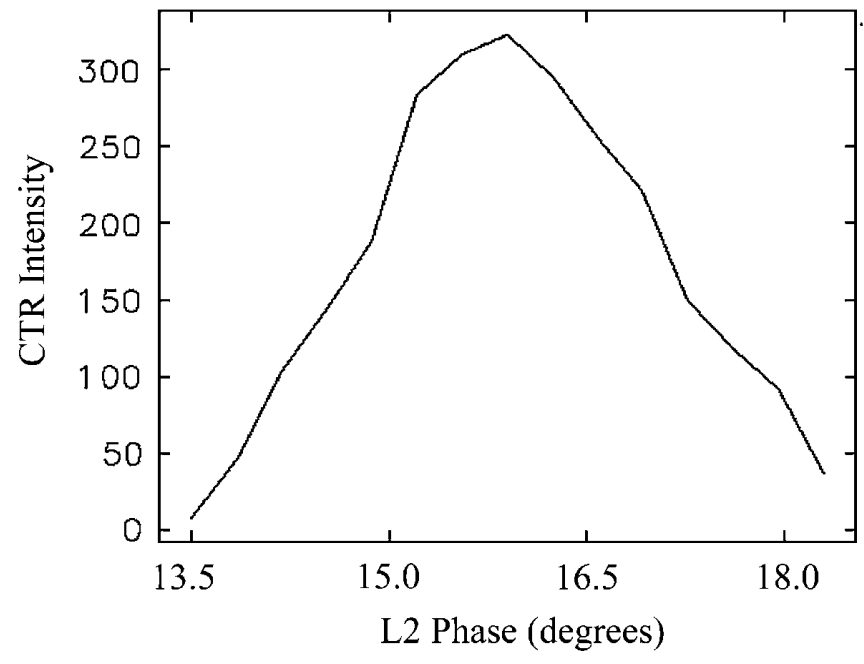

FIG. 2. Variation of FIR CTR signal from the Golay cell with the upstream L2 rf accelerator phase setting.

at zero position) as shown in Fig. 2. A very strong variation of the FIR CTR signal with L2 phase was observed. There was almost no CTR signal seen when the beam was uncompressed, and at peak compression 300 units were observed. Subsequent autocorrelation scans showed a profile width of $\sim 65 \mu \mathrm{m}$ (FWHM) as seen in Fig. 3. Two separate scans are displayed in the plot. This would mean a path length difference of $130 \mu \mathrm{m}$ between the mirror positions, or about $430 \mathrm{fs}$ (FWHM). The initial photocathode drive laser pulse was 3 to 4 ps (FWHM) as determined by an optical autocorrelator.

Having determined we had full compression from the Golay-cell data, we then sampled the beam images at the three screens after the chicane in the first emittancemeasurement area. The samples shown are from FS5, the

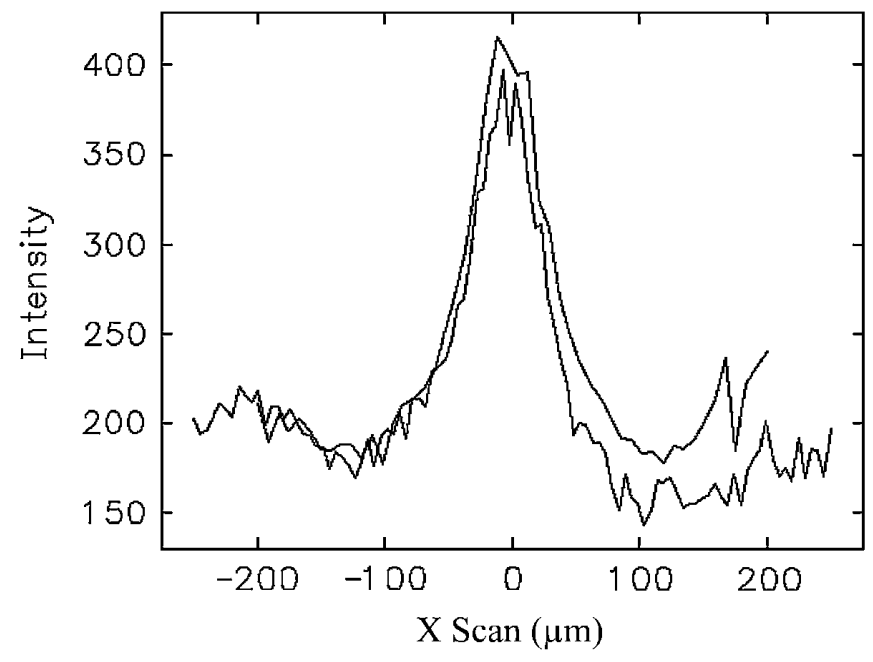

FIG. 3. FIR CTR autocorrelation performed after the chicane with a near maximum compression setup for the PC gun. The two scan profile widths are about $65 \mu \mathrm{m}$ (FWHM) corresponding to a path length difference of $130 \mu \mathrm{m}$ between the mirror positions. 

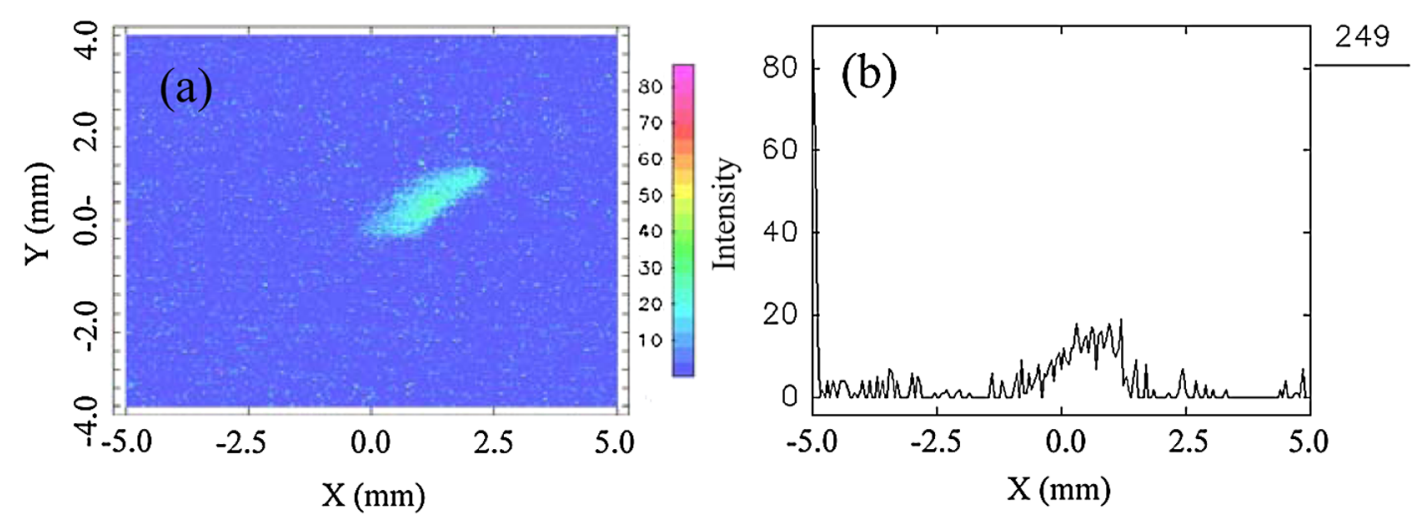

FIG. 4. (Color) OTR beam image (a) and horizontal profile (b) sampled through distribution after BC with L2 phase = 12 degrees, close to minimum compression.
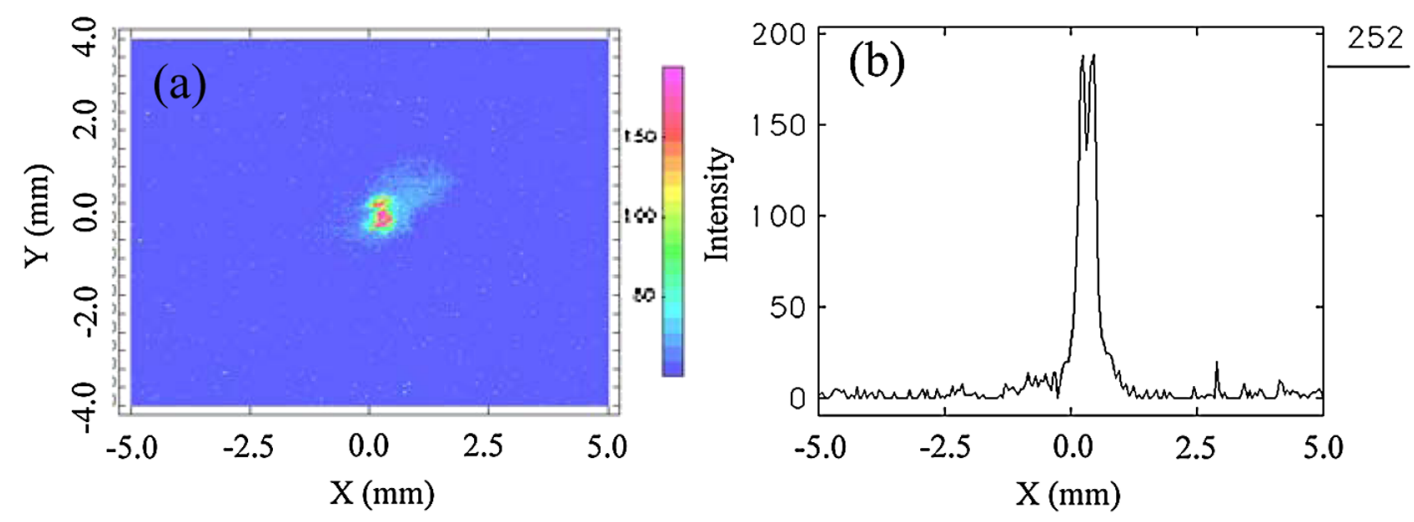

FIG. 5. (Color) OTR beam image (a) and horizontal profile (b) through a spike in distribution after BC with L2 phase $=14.9$ degrees, close to maximum compression.

third screen of the set. At the point of low compression the OTR image is weak. No sharp features are seen as shown in Figs. 4(a) and 4(b). The profile is sampled a little off center of this image at row 249, but it is representative of its flatness and low intensity of 15-25 counts. In contrast, the image taken near full compression as indicated by the FIR CTR signal has significantly enhanced localized spikes of about $250 \mu \mathrm{m}$ (FWHM) extent as shown in Fig. 5. The pseudocolor intensity scale at the right of the image shows that the red areas are high intensity. The profile at row 252 in Fig. 5(b) shows the peak intensity of 180 counts, almost 10 times the adjacent intensities in the beam-image footprint. On this particular run, we noted that there seemed to be a preferred location in the footprint to be enhanced. The enhanced vertical band in Fig. 5(a) is the same area that shows lower enhancements with less compression.

An additional indication of the effect is shown in Fig. 6, where the peak intensities from the vertical profiles taken through the images at each phase setting show the rapid increase in the enhanced OTR signal in just a few degrees of L2 phase change. An order of magnitude enhancement is clearly seen in this plot. A similar plot for horizontal profiles was done, and this showed a slight shift of the intensity peak compared to the CTR data by 0.4 degrees in
L2 phase. Integrated intensities of the ten images at each phase setting do not show the same degree of enhancement. This analysis shows 1.6 to 2 times larger integrated counts at peak compression compared to the uncompressed case,

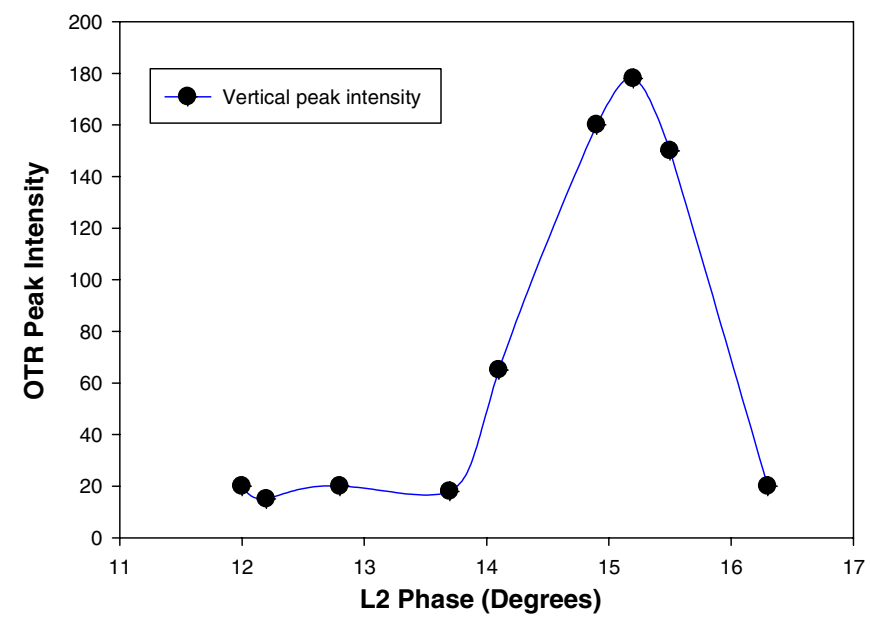

FIG. 6. (Color) Plot of the peak intensities from the OTR image vertical profiles taken near the enhanced regime coordinates as a function of L2 phase in degrees. 


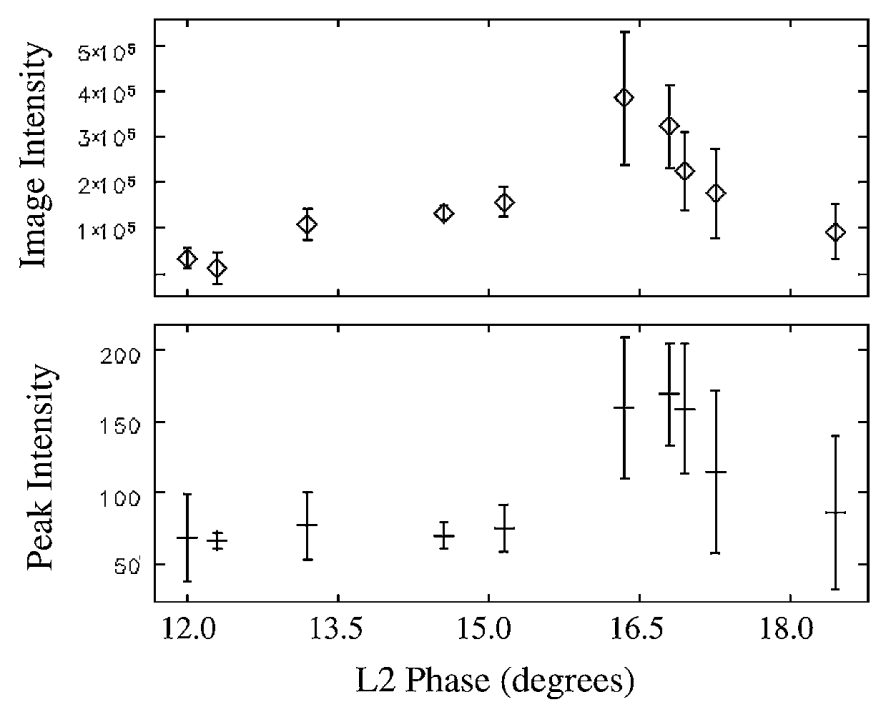

FIG. 7. Plot of the OTR image integrals (top) and OTR peak intensities (bottom) versus L2 phase (degrees). The enhancements occur near the FIR CTR signal maximum.

with larger fluctuations in the compressed conditions as shown in Fig. 7 (top). The peak intensities on average increase by a factor of 2-3 at the point of maximum compression as shown in the lower plot of Fig. 7. The error bars are the standard deviation of the parameters for the images. Since charge transport was preserved to $10 \%$ during the phase scan and CSR-induced transverse clumping of electrons would not result in the integrated image intensity integrals' increasing, the enhanced-signals results are consistent with a coherent enhancement of some kind related to the longitudinal distribution.

We accelerated the beam to $375 \mathrm{MeV}$ and imaged the beam spot with OTR at a downstream station, Sta-5. As described previously, this station included transport of the signal outside of the tunnel to a small optics lab. First, we

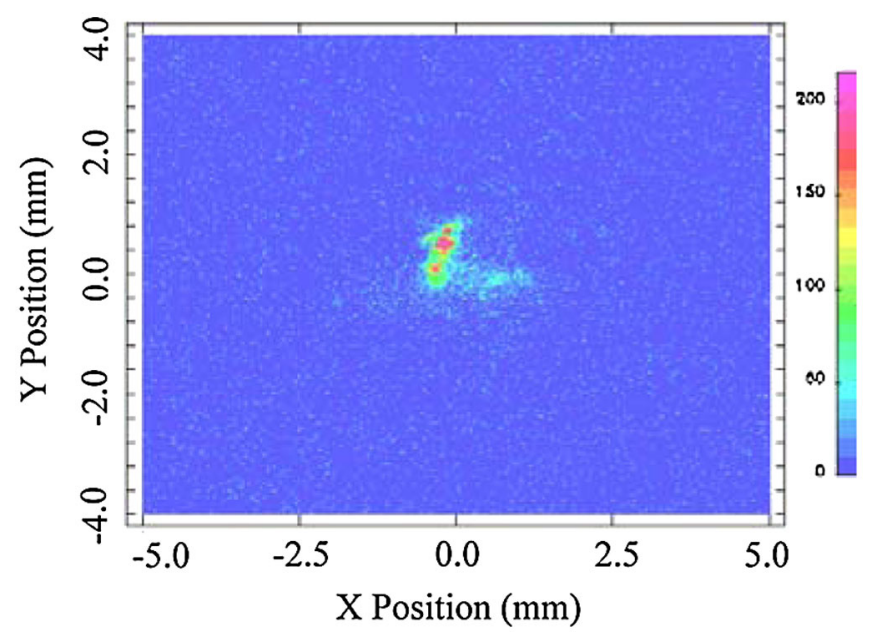

FIG. 8. (Color) OTR image at $375 \mathrm{MeV}$ showing the enhancements are still present after acceleration beyond the bunch compressor.
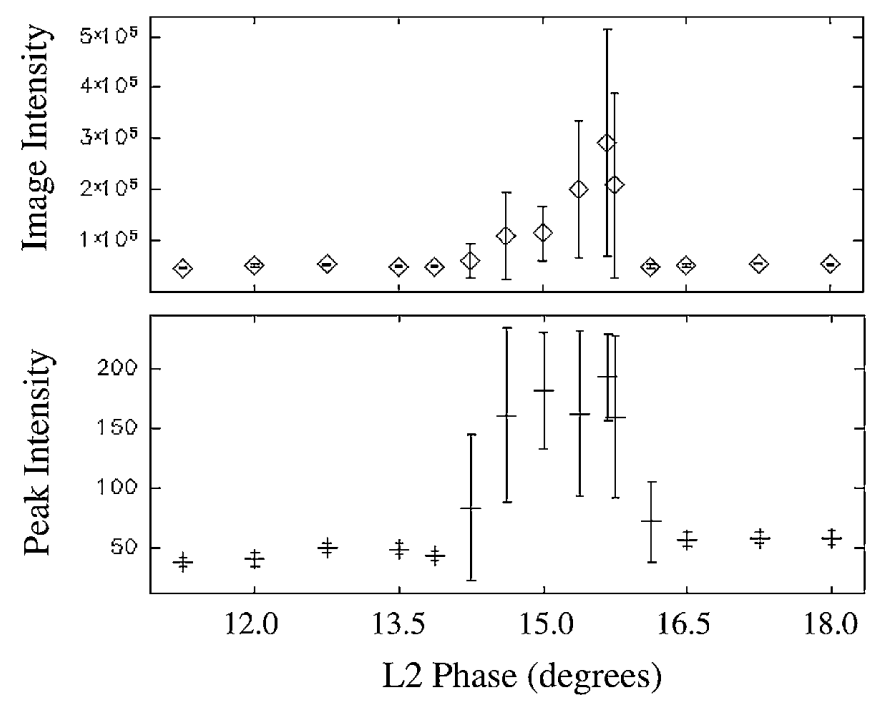

FIG. 9. Plot of the OTR image intensity integrals (top) and OTR peak intensities (bottom) at $375 \mathrm{MeV}$ using automated postprocessing versus L2 phase. The OTR enhancements occur near the same phase as the observed FIR CTR signal maximum.

still see enhanced localized spikes when the beam is compressed as shown in Fig. 8. We also confirmed that these spikes were present at a compression level corresponding to $1 / 2$ of the peak CTR signal, although their intensity varied more from shot to shot. In Fig. 9 we show the processed image intensity integrals (top) and the peak intensities (bottom) versus the L2 phase set point at this location. In this case enhancements of the OTR signals when at maximum compression are about 3-6 times the normal intensity. The strong fluctuations of these enhanced areas are suggestive of a coherent process seeded by noise. [We recall that previously our self-amplified spontaneous emission (SASE) free-electron laser (FEL) experiments indicated preferred hot spots in the spatial distribution of the beam [15].]

\section{COTR SPECTRAL EFFECTS}

In order to assess the spectral dependency of the OTR enhancements, we accelerated the beam to $325-375 \mathrm{MeV}$ and imaged the beam spot with OTR at Sta-5. At full compression, we checked the spectral dependency of the enhancements by inserting the bandpass filters in front of the CCD camera. Our preliminary results were that the enhancements were seen at all central wavelengths from 400 to $750 \mathrm{~nm}$ (in steps of $50 \mathrm{~nm}$ ), although relatively weaker in the 400 to $500-\mathrm{nm}$ regime than at $550 \mathrm{~nm}$. We checked the spectral dependence of incoherent OTR from the TC gun beam and saw an intensity roll-off in this short wavelength interval which we attributed to the CCD camera response to these different wavelengths. However, this roll-off was not as much as the observed COTR intensity roll-off [16]. In Fig. 10 we show the image intensities normalized for CCD response and beam charge. The over- 


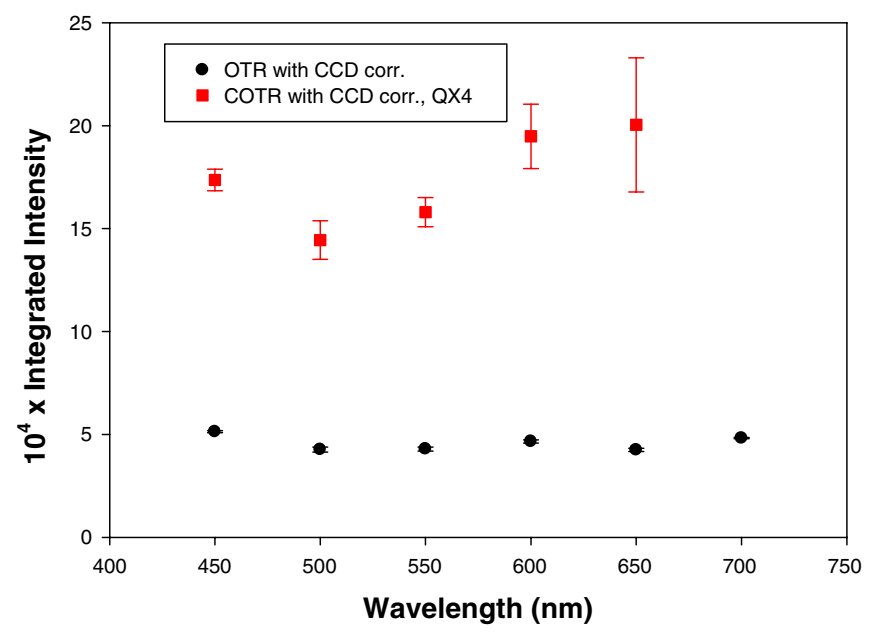

FIG. 10. (Color) Evaluation of the OTR and COTR image intensities versus the bandpass filter center wavelength for the compressed PC rf gun beam (red squares) and compressed TC rf gun beam (black circles) with charge normalized. The COTR has a relatively stronger red component than the incoherent OTR. all enhancement of the COTR from the PC gun beam is 3 to 4 times the OTR from the TC gun beam per unit charge, and the COTR has more red enhancements.

This is corroborated by the spectrometer measurements shown in Fig. 11 where the $x$-localized emission point for COTR is shown in each image. The scintillators show the full $\sim 2$-mm width of the $x$ beam spot, while the COTR source intensity and $x$ locations vary. It is noted that both the YAG:Ce screen and LSO:Ce screen are normal to the beam with an $\mathrm{Al}$ mirror behind each at 45 degrees. This mirror is in the same plane as the Al OTR screen when it is inserted. These three options are on three different actuators. The COTR spectral streak profile is more broadband than the YAG:Ce spectrum which is centered near $530 \mathrm{~nm}$ and the LSO:Ce spectrum which is centered near $415 \mathrm{~nm}$ as shown in Fig. 11. Such results indicate that this latter scintillator-mirror configuration would still result in COTR spikes in the $x-y$ images. These results support the mitigation scheme described in the next section.

We next extended our spectral studies into the near infrared (NIR) by installing a GaAs photocathode micro-
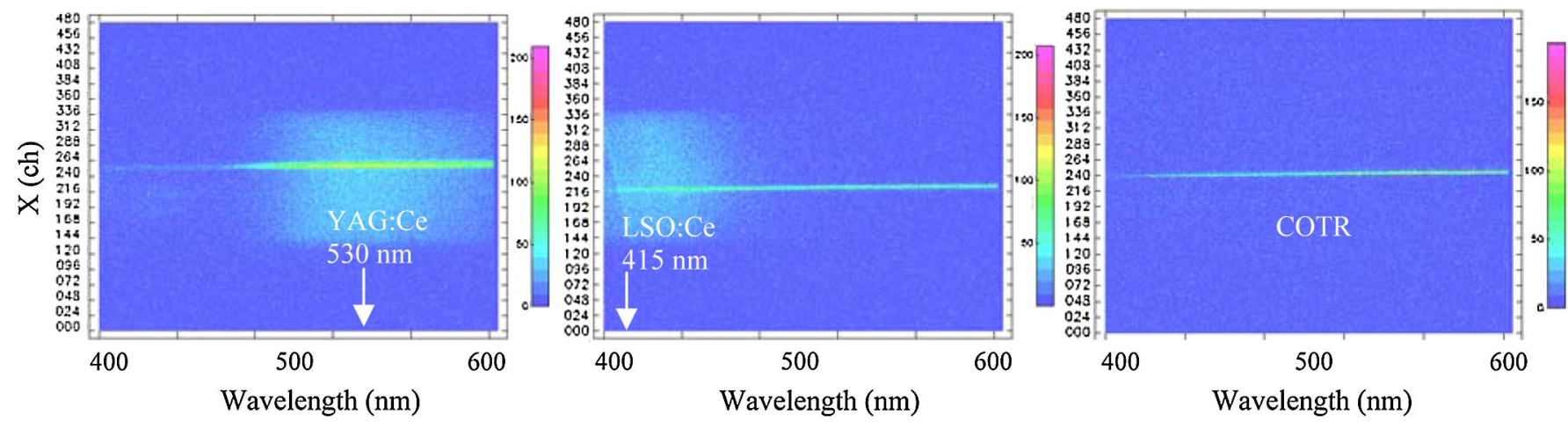

FIG. 11. (Color) Imaging spectrometer results with CCD camera readout in $x$ - $\lambda$ space for YAG:Ce plus an Al mirror (left), LSO:Ce plus an Al mirror (middle), and an Al mirror only (right). The localized $x$ extent of the COTR streak in wavelength is evident in all three configurations.
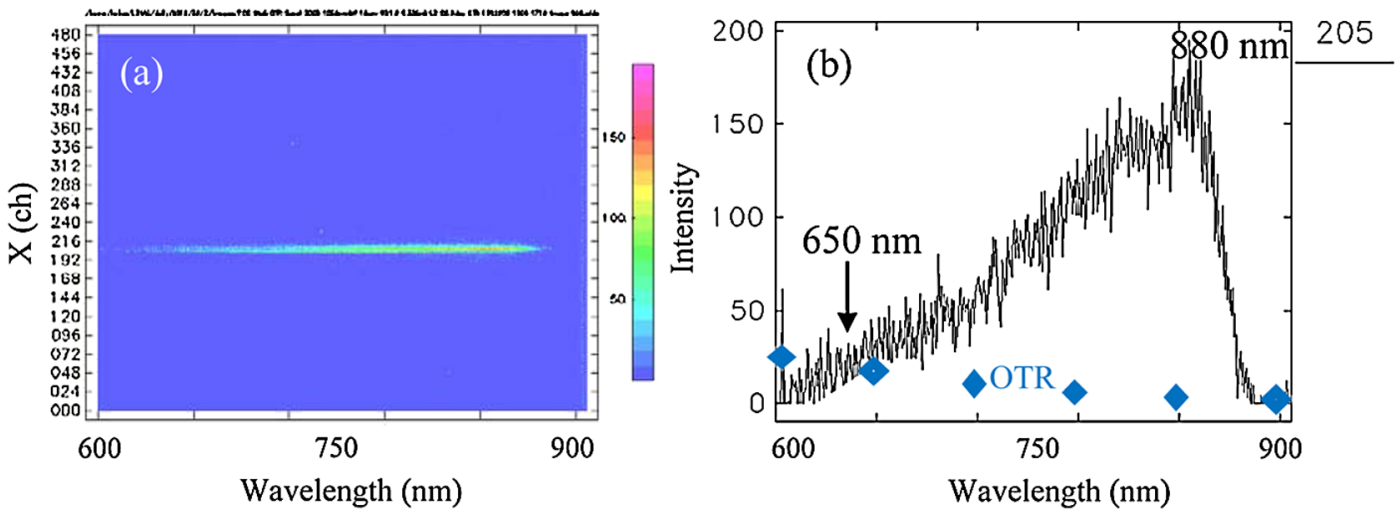

FIG. 12. (Color) COTR spectrum with NIR MCP utilized showing (a) $x$ - $\lambda$ image and (b) profile through the localized $x$ enhancement. The entrance slit was narrowed to give few-nm resolution with a 95 lines per mm grating. In addition, an incoherent OTR broadband spectrum is indicated by the blue diamonds. 
channel plate (MCP)-intensified CCD camera (Pulnix DN007) at the spectrometer output port. This photocathode has almost constant quantum efficiency from 525 to $880 \mathrm{~nm}$, and thus complements the spectral response of the bare CCD camera whose sensitivity decreases strongly from 600 to $880 \mathrm{~nm}$. As seen in Fig. 12, the COTR is climbing in intensity dramatically from 650 to $880 \mathrm{~nm}$. In this particular run, the compression was adjusted more strongly by increasing the gradient of the L2 rf section, and the autocorrelator gave a 30\% narrower width. These are the first spectral data showing the visible red wavelengths and NIR wavelengths together. In this particular pulse and $x$ location, the intensity has dropped to a gain factor near one at $600 \mathrm{~nm}$ when compared to incoherent OTR at $600 \mathrm{~nm}$. We obtained spectra with the TC gun beam in a separate run with $2 \mathrm{nC}$ in the macropulse using the same grating and grating offset, and we observe the $1 / \lambda^{2}$ like dropoff of the OTR signal (times $1 / 4$ for charge normalization to the PC gun micropulse) as indicated by the diamonds in Fig. 12(b).

\section{MITIGATION OF COTR}

With the strong evidence of the COTR from the LSCIM being generally more enhanced in the red end of the spectrum at APS and LCLS, we pursued several mitigation concepts. Supported both by the bandpass filter tests where we could not process net counts for the $400 \mathrm{~nm} \times 40 \mathrm{~nm}$ filtered COTR and the spectrometer tests at APS, we demonstrated the use of such a $400 \mathrm{~nm} \times 40 \mathrm{~nm}$ bandpass filter to preferentially transmit the incoherent OTR signals while blocking most of the COTR seen in Fig. 13(a). With our eight-bit analog camera we could not image the 0.4-nC beam with the filtered OTR so we also employed the LSO: Ce crystal which has its peak emissions near $415 \mathrm{~nm}$ [17] as a means to enhance beam-image signal at low charge as seen in Fig. 13(b). However, the reported sensitivity of the 12-bit digital camera used at LCLS [18] would have been sufficient for a beam image to be obtained with the filtered OTR and $300 \mathrm{pC}$ of charge. The LSO:Ce scintillator efficiency is similar to that of YAG:Ce, but it has a faster response time as indicated in Table II.

We have evaluated the advantages of the violet end of the spectrum that has generally more OTR than COTR for the LCLS case as shown in Fig. 14. We used a simple model for the $1 / \lambda^{2}$ dependence of the OTR spectral content, and we compared this curve to the COTR estimated by multiplying the OTR intensity by the enhancement/gain factor based on the published model's match to experimental spectral data at LCLS at a screen located after their first chicane [8]. The calculated spectrum for a $3-\mathrm{keV}$ slice energy spread matched reasonably well the experimental spectrum reported in that study. We then overlaid the CCD camera response on these curves to show which wave-
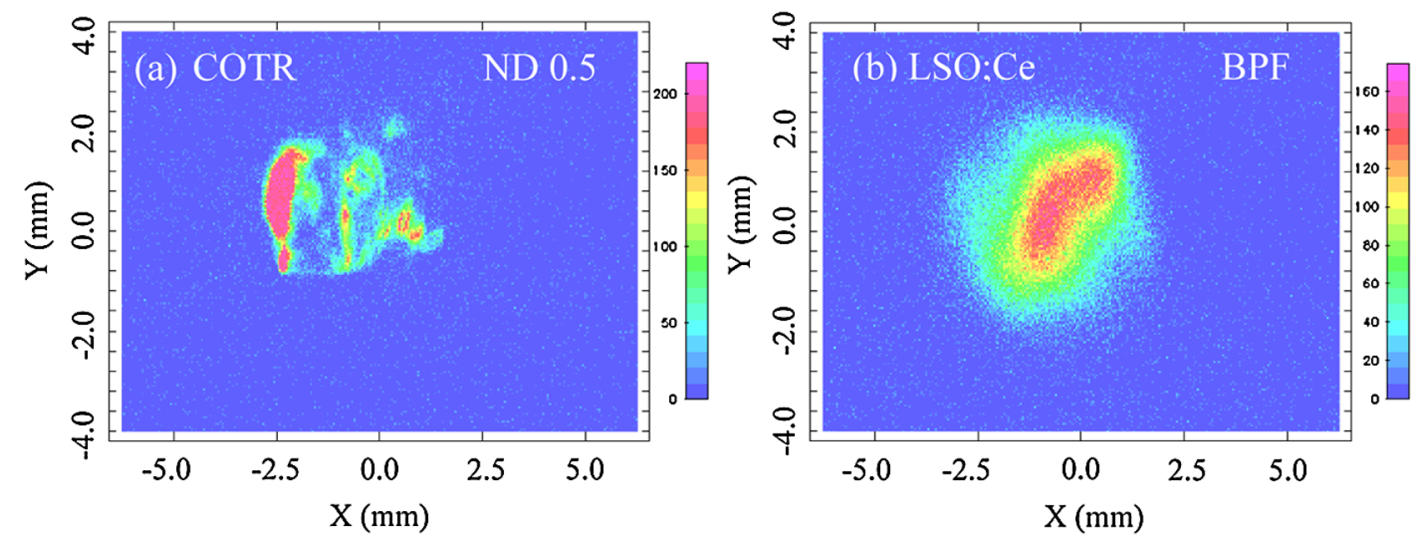

FIG. 13. (Color) Comparison of beam images at $325 \mathrm{MeV}$ at APS from (a) COTR and (b) with mitigation of COTR using the $400 \mathrm{~nm} \times 40 \mathrm{~nm}$ bandpass filter (BPF) and LSO:Ce crystal.

TABLE II. Summary of some radiation converter screen properties for Ce-doped scintillators and an Al OTR screen.

\begin{tabular}{lcccc}
\hline \hline Converter & $\begin{array}{c}\text { Spectrum } \\
(\text { FWHM })^{\mathrm{a}}, \text { peak }\end{array}$ & Efficiency & $\begin{array}{c}\text { Response time } \\
(\text { FWHM })\end{array}$ & Comment \\
\hline YAG:Ce & $487-587,526 \mathrm{~nm}$ & $1.0^{\mathrm{a}}$ & $89 \mathrm{~ns}^{\mathrm{a}}$ & $460 \mu \mathrm{m}$ thick \\
LSO:Ce & $380-450,415 \mathrm{~nm}$ & $0.46^{\mathrm{a}}$ & $40 \mathrm{~ns}^{\mathrm{a}}$ & $530 \mu \mathrm{m}$ thick \\
YAP:Ce & $350-400,369 \mathrm{~nm}$ & $\sim 0.5$ & $28 \mathrm{~ns}$ & $460 \mu \mathrm{m}$ thick \\
OTR & Broadband & $0.0013^{\mathrm{a}}$ & $\sim 10 \mathrm{fs}$ & Surface \\
\hline \hline
\end{tabular}

${ }^{\mathrm{a}}$ Values from Ref. [17]. 


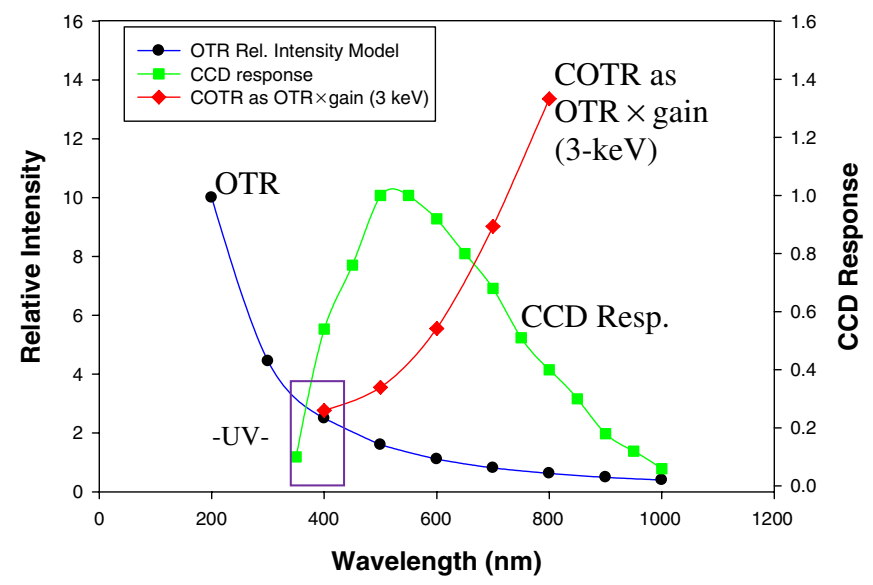

FIG. 14. (Color) Plots of the nominal OTR intensity dependence on $1 / \lambda^{2}$, the COTR intensity using the calculated gain factor at LCLS for a 3-keV slice energy spread [8], and the nominal CCD camera relative response. The small violet rectangle indicates the $400 \mathrm{~nm} \times 40 \mathrm{~nm}$ BPF regime next to the UV.

lengths were detected. The violet box indicates the $400 \mathrm{~nm} \times 40 \mathrm{~nm}$ bandpass filter (BPF) regime and graphically shows the COTR mitigation compared to using all of the CCD's response regime. The APS COTR case is less violet in hue since we barely detect on the monitor some weak spikes when we use the $400 \mathrm{~nm} \times 40 \mathrm{~nm}$ filter, so the mitigation should be strong. We proposed extending the concept by imaging in the ultraviolet $(380-200 \mathrm{~nm}$ ), a regime where the COTR should be relatively even more reduced, i.e., where the gain factor is near 1.0. There is a trade-off, in that standard UV-sensing cameras have reduced sensitivity in the visible regime. However, our present lens transport at Sta-5 does not transmit UV so another experimental setup would be needed. The mitigation options are as follows: (i) use BPF at $400 \mathrm{~nm}$ or short pass filter (SPF) to select preferentially OTR over COTR; (ii) use BPF centered at 400 or SPF with an LSO:Ce scintillator to image lower charges; (iii) use solar blind or UV filters with UV-sensing cameras to select 380 to $200 \mathrm{~nm}$ OTR with minimal COTR (as schematically shown in Fig. 14).

\section{CTR AND OTR RESULTS WITH TC RF GUN BEAM}

In the course of our studies, we decided to test the effect with the TC rf gun beam. In this case we could generate about $40 \mathrm{pC}$ per micropulse in an 8-ns long macropulse of 23 micropulses that contained about $1 \mathrm{nC}$ total. So the integrated OTR signal should be similar to that of the PC gun beam. We also have no drive laser involved. With the combination of compression in the alpha magnet of the TC gun system and the chicane, we were able to generate an autocorrelation profile width of $\sim 62 \mu \mathrm{m}$ (FWHM) as shown in Fig. 15. This result is very similar to the PC gun result in Fig. 3, so we have about a sub-0.5 ps FWHM

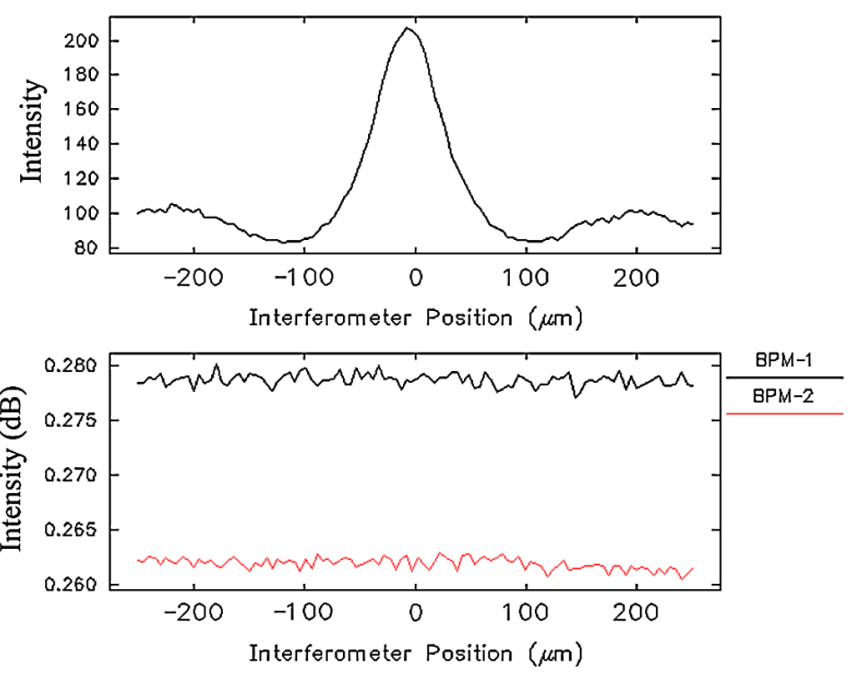

FIG. 15. (Color) FIR CTR autocorrelation performed after the chicane near maximum compression setup for the TC gun. The scan profile width (top) is about $62 \mu \mathrm{m}$ (FWHM). The BPM sum signals (bottom) from positions after the chicane were also tracked and show little change during the scan.

micropulse at a beam energy now of $150 \mathrm{MeV}$. The main difference after the chicane is that there is $\sim 10$ times less charge in the micropulse compared to the PC gun beam and 10 times lower peak current. One would expect a significant reduction in any coherent mechanisms. We observed no localized OTR enhancements at the screen after the chicane, nor at the downstream location Sta-5 at the end of the linac. The integrated intensities were the same to about $10 \%$ throughout the L2 phase scan. An image from the latter screen under full compression conditions is shown in Fig. 16. We repeated the spectral intensity measurements with the filters and determined the nominal overall response of the system to the OTR spectrum and the CCD sensitivity factors as shown in Fig. 10.

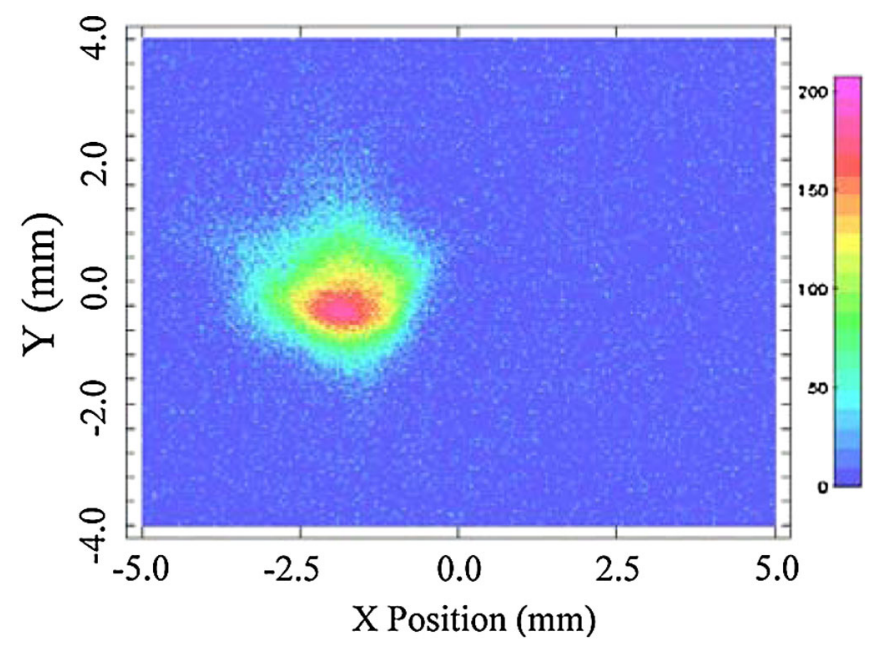

FIG. 16. (Color) An example OTR image of the TC $\mathrm{rf}$ gun beam at the $375-\mathrm{MeV}$ station with maximum compression in the chicane. No localized spatial spikes are evident. 


\section{DISCUSSION}

We have verified the existence of the enhanced OTR effect after compression at APS and characterized its variation with compression. We accelerated this beam through L4 and L5 and found the enhancement persists and is even stronger. We performed base spectral measurements using bandpass filters and a spectrometer. We also compressed a TC gun beam at much lower micropulse charge (and with a larger energy spread) and observed no resulting COTR. Several characteristics of the enhanced OTR signals may be used to evaluate which mechanism is involved. If we consider transport efficiency during the phase scan, photon number enhancements, intensity fluctuations, charge dependence, and spectral structure observed, we can discriminate among LSC microbunching, a longitudinal spike, multiple submicron spikes, and CSRinduced transverse clumping. We initially considered whether there were microbunching from the drive laser interaction with the low-energy photoelectrons such as found under high laser power conditions [19], but a simple calculation of the laser field strengths showed this effect would not be measurable. Since we measured that the transported charge through the chicane was constant to $10 \%$ during the phase scan, we cannot explain the large photon number enhancements as only due to CSR-induced transverse clumping of the charge distribution. Both our preliminary spectral sampling across the visible light region with bandpass filters of 40-nm bandwidths and an imaging spectrometer showed a broadband character like that of incoherent OTR, but COTR had a relatively stronger red component. Our broadband visible spectrometer data are consistent with coherent enhancements of wavelengths longer than a fine longitudinal structure, such as a leadingedge spike or multiple fine spikes, but in addition they are consistent with the broadband LSCIM based spectra. We have reported separately that we have found a connection with the localized enhancements seen in our old SASErelated COTR data at $530 \mathrm{~nm}$ and with the apparent LSCIM beam structures entering the undulators [15]. The localized spatial spikes of COTR are nominally consistent in transverse size with the coherence length of $\sim 1.5 \gamma \lambda / 2 \pi$ for visible wavelengths such as discussed by Venturini [20]. There is also now clear evidence from the laser heater tests at LCLS that the visible light COTR is strongly damped when the slice energy spread is purposely increased, as expected [21]. These latter two aspects support the LSCIM interpretation for the COTR after the APS compressor which is similar in design to LCLS's.

\section{SUMMARY}

In summary, we have initiated investigations on the enhancement of OTR signals in the visible light regime following bunch compression of our PC $\mathrm{rf}$ gun beam at APS. Although the enhancements are not as high as that reported at LCLS $[9,10]$, we do see order of magnitude signal increases in localized spatial spikes within the images after the chicane and 2 orders of magnitude signal enhancements at the end of the linac. At this time the coherent enhancement appears consistent with many fine submicron spikes or LSCIM at visible wavelengths after bunch compression. The practical effect is that one cannot simply evaluate emittance of the beam for different compressions using the OTR screen images. One normally uses the YAG:Ce screens at APS for signal considerations, and LSO:Ce screens are now an option to consider. We did not see the effects in the TC gun beam when it was compressed similarly. Since this involved equally short electron pulses but only $40 \mathrm{pC}$ per micropulse, it lends support to the conclusion that the enhancement is driven by a coherence phenomenon. We have clearly established the COTR trend toward higher intensities at the red end of the visible spectrum and into the NIR so that mitigation schemes based on imaging the violet and ultraviolet end of OTR are appropriate at APS and LCLS, and similar accelerator configurations. The growing interest in these COTR effects is indicated by the time allowed for discussion in the recent microbunching instability workshop [22].

\section{ACKNOWLEDGMENTS}

The authors acknowledge support from M. Wendt of Fermilab and R. Gerig, K.-J. Kim, and H. Weerts of the Argonne Accelerator Institute. They acknowledge S. Shoaf (ANL) for controls support and K. Nemeth (ANL) for estimating the laser-electron interaction. They also acknowledge discussions on COTR with D. Dowell, H. Loos, J. Frisch, and Z. Huang of LCLS and with R. Fiorito of the University of Maryland and D. Rule of NSWC. This work was supported by U.S. Department of Energy, Office of Science, Office of High Energy Physics, under Contract No. DE-AC02-06CH11357.

[1] Proceedings of the ICFA 17th Advanced Beam Dynamics Workshop on Future Light Sources (Argonne National Laboratory, Argonne, IL, 1999), and other workshops in the series.

[2] S. V. Milton et al., Science 292, 2037 (2001).

[3] Linac Coherent Light Source (LCLS) Conceptual Design Report, SLAC Report No. SLAC-R-593, 2002.

[4] M. Borland et al., in Proceedings of the Particle Accelerator Conference, Chicago, IL, 2001 (IEEE, New York, 2001), pp. 2707-2709; M. Borland et al., Nucl. Instrum. Methods Phys. Res., Sect. A 483, 268 (2002).

[5] E. L. Saldin, E. A. Schneidmiller, and M. V. Yurkov, Nucl. Instrum. Methods Phys. Res., Sect. A 490, 1 (2002).

[6] E. L. Saldin, E. A. Schneidmiller, and M. V. Yurkov, DESY Report No. TESLA-FEL-2003-02, 2003.

[7] Z. Huang et al., Phys. Rev. ST Accel. Beams 7, 074401 (2004).

[8] D. Ratner, A. Chao, and Z. Huang, in Proceedings of FEL08, Gyeongju, Korea, 2008, JACoW, TUPPH041 (to be published). 
[9] D. H. Dowell et al., in Proceedings of FEL07, Novosibirsk, 2007, JACoW, WEAAU01; R. Akre et al., Phys. Rev. ST Accel. Beams 11, 030703 (2008).

[10] H. Loos et al., Proceedings of FEL08, Gyeongju, Korea, 2008, JACoW, THBAU01 (to be published).

[11] M. Borland, Phys. Rev. ST Accel. Beams 4, 074201 (2001).

[12] A. H. Lumpkin et al., Phys. Rev. Lett. 86, 79 (2001).

[13] A. H. Lumpkin et al., in Proceedings of FEL05, eConf C0508213, 608 (2005).

[14] B. Yang et al., AIP Conf. Proc. 648, 393 (2002).

[15] A. H. Lumpkin, R. J. Dejus, and N. S. Sereno, Proceedings of FEL08, Gyeongju, Korea, 2008, JACoW, THBAU05 (to be published); Phys. Rev. ST Accel. Beams 12, 040704 (2009).
[16] A. H. Lumpkin, N. S. Sereno, W. J. Berg, M. Borland, Y. $\mathrm{Li}$, and S. Pasky, Proceedings of LINAC08, Victoria, Canada, 2008, JACoW (to be published).

[17] W. J. Berg, A. H. Lumpkin, and B. X. Yang, in Proceedings of the XX International Linac Conference, Monterey, CA, 2000, MOC06, p. 158.

[18] H. Loos (private communication).

[19] Karoly Nemeth et al., Phys. Rev. Lett. 100, 095002 (2008).

[20] M. Venturini, Phys. Rev. ST Accel. Beams 11, 034401 (2008).

[21] P. Emma (private communication).

[22] Agenda for Microbunching Instability Workshop II, Chairman W. M. Fawley, LBNL, Berkeley CA, 2008. 